\title{
ANALYZING UNSTRUCTURED DATA: TEXT ANALYTICS IN JMP
}

\author{
$\underline{\text { Volker Kraft }}$ \\ SAS Institute - JMP Division, JMP Academic Team \\ volker.kraft@jmp.com
}

As much as $80 \%$ of all data is unstructured but still has exploitable information available. For example, unstructured text data could result from comment fields in surveys or incident reports. You want to explore this unstructured text to better understand the information that it contains. Text Mining, based on a transformation of free text into numerical summaries, can pave the way for new findings.

This example of the new text mining feature in JMP starts with a multi-step text preparation using techniques like stemming and tokenizing. This data curation is pivotal for the subsequent analysis phase, exploring data clusters and semantics. Finally, combining text mining results with other structured data takes familiar multivariate analysis and predictive modeling to a next level.

\section{INTRODUCTION}

In the digital world of today, the cost and the limitation barriers in storing and accessing data have been pretty much removed. In 2015, IDC Research estimated that $90 \%$ of this vast amount of digital data is unstructured data, including responses to open-ended survey questions, social media, email, maintenance reports and HTML web pages (Vijayan, 2015).

The new Text Explorer platform in JMP 13 does not only allow to learn from unstructured text data without programming, but can make the tedious cleanup of inherently messy text data quick, easy, and fun. As soon as the data are ready for analysis, you are typically interested in questions like

- Can we identify groups of documents that are similar to one another as a way of summarizing content or uncover themes?

- Are there any unusual documents ("outliers")?

- Can we discover relationships between structured and unstructured data?

- Can we improve predictive models by combining text data with other data sources?

We all know how to tackle these questions with more traditional, structured data. Text exploration in JMP amounts to transforming text data into a more traditional rectangular data format, which then supports classical multivariate analysis techniques like regression, CART or GLM.

It is amazing to see how much you can learn from text data just by using "simple" data mining techniques, without worrying about higher linguistic levels like syntax analysis or natural language understanding. Text Explorer is typical JMP and makes text analytics easily accessible, very interactive and visual. Statistical discoveries from unstructured text data can also be a lot of fun, especially in teaching data mining or multivariate analysis courses.

Let us introduce the basic terminology and workflow of text analytics in JMP, by looking at a real-world example.

\section{BASIC TERMINOLOGY AND WORKFLOW}

The JMP table "NTSBAccidentReports2001-2003.jmp" (Fig. 1) provides data about 3,235 accidents in air traffic, collected between 2001-2003 by the National Transportation Safety Board in the US. One column, 'narr_cause', is formatted as "unstructured text". This column is called a corpus, and each cell stores a document. One task in this example is to explore relationships between the (unstructured) reports and other (structured) information like month of year or weather conditions incl. temperature and wind. Another goal is predicting fatality (YES/NO) of an accident based on the text information stored in accident reports. 


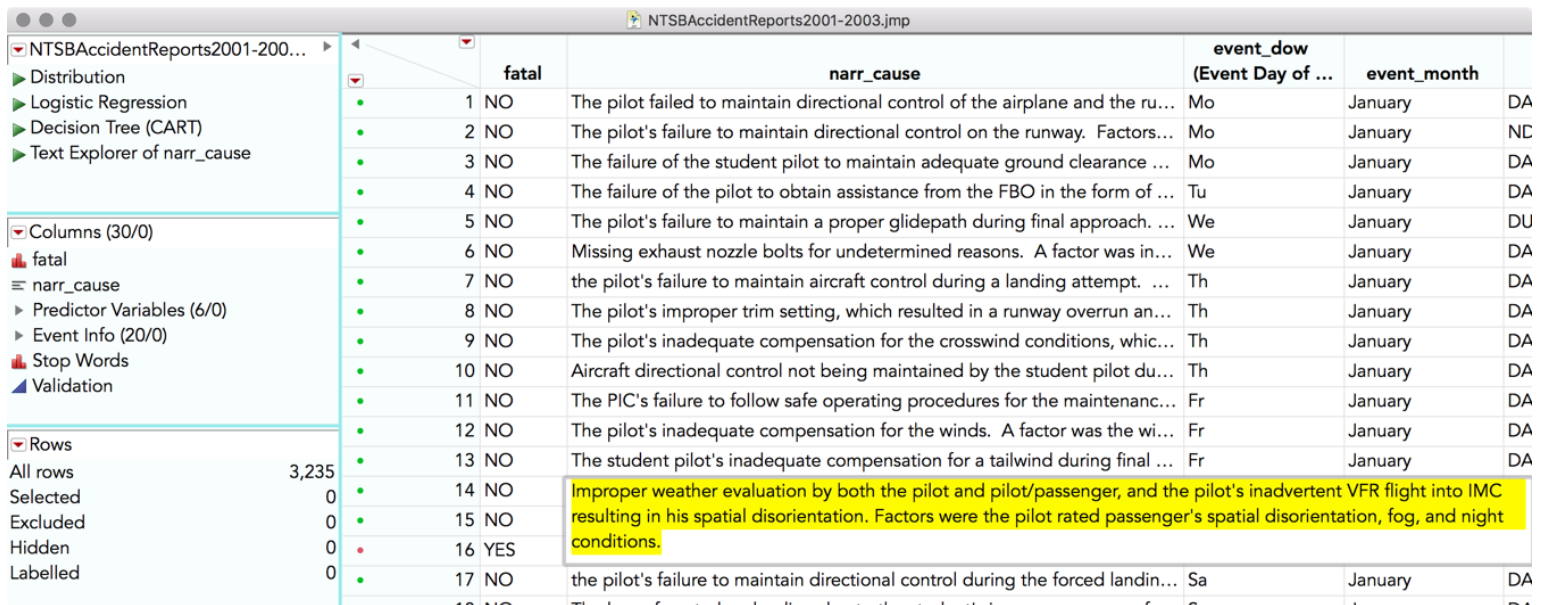

Fig. 1: NTSB Accident Reports, with unstructured text column 'narr_cause' (=corpus), containing 3,235 cells (=documents)

According to Klimberg et al. (2016) the process of text analysis can be split into three phases:

1. Term Creation: This phase does all text cleanup and develops the so-called document term matrix (DTM). The DTM is a set of indicator variables that represent the terms (columns) in the documents (rows). Terms are all words and phrases which will be considered in the analysis phase. Techniques like tokenizing, phrasing and terming are used to initially develop the DTM. Subsequently, you explore the set of variables and curate the DTM, by grouping words or removing infrequent words, until you are satisfied.

2. Text Analysis: Text visualization and the text multivariate techniques of clustering, principal components, and factor analysis are used to understand the composition of the DTM.

3. Explore relationships and predict outcomes: If a dependent variable exists (here: 'fatal'), you can use the text multivariate analysis results, along with other structured data, as independent variables in a predictive technique.

\section{PART I: TERM CREATION}

We launch Text Explorer from the Analyze menu in JMP and select 'narr_cause' as the Text Column for analysis (Fig. 2). All other settings are (carefully preconfigured) defaults. Both stemming and regular expressions for tokenizing are advanced options and not used here.

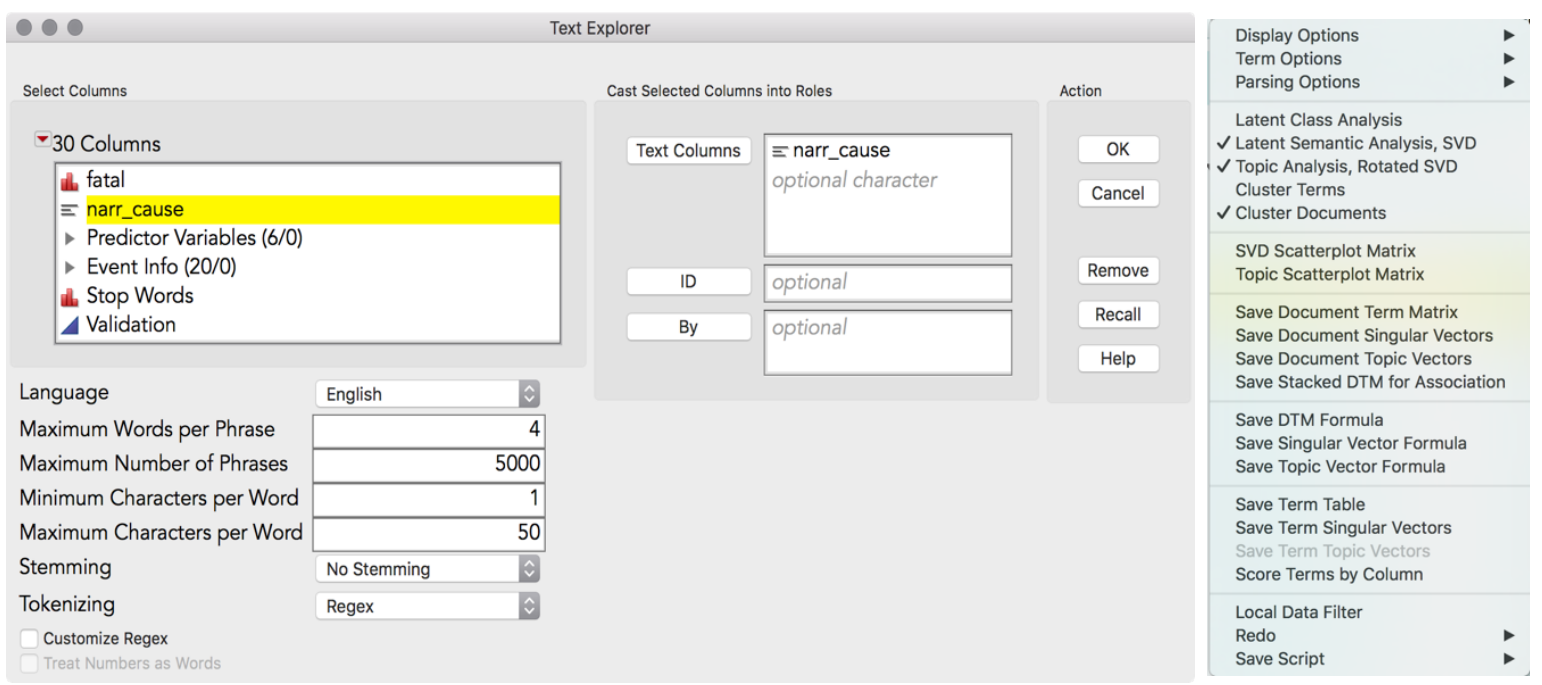

Fig. 2: Launching Text Explorer to analyze text column 'narr_cause' (left).

Top red triangle menu in Text Explorer report (right). 
The summary in Fig. 3 tells us that 3235 documents were parsed into 85675 tokens, representing 1328 different terms. The terms used most often are 'landing' and 'failure'. Some terms were manually removed from the term list (right-click > "Add Stop Word").

Phrases are word sequences which occur more than once in the corpus. The phrase used most often is 'failure to maintain', occurring 844 times and consisting of three words. The grey phrases have been added to the term list (right-click > "Add Phrase"), since they represent important concepts. 'Landing gear' in red was already added as a system phrase.

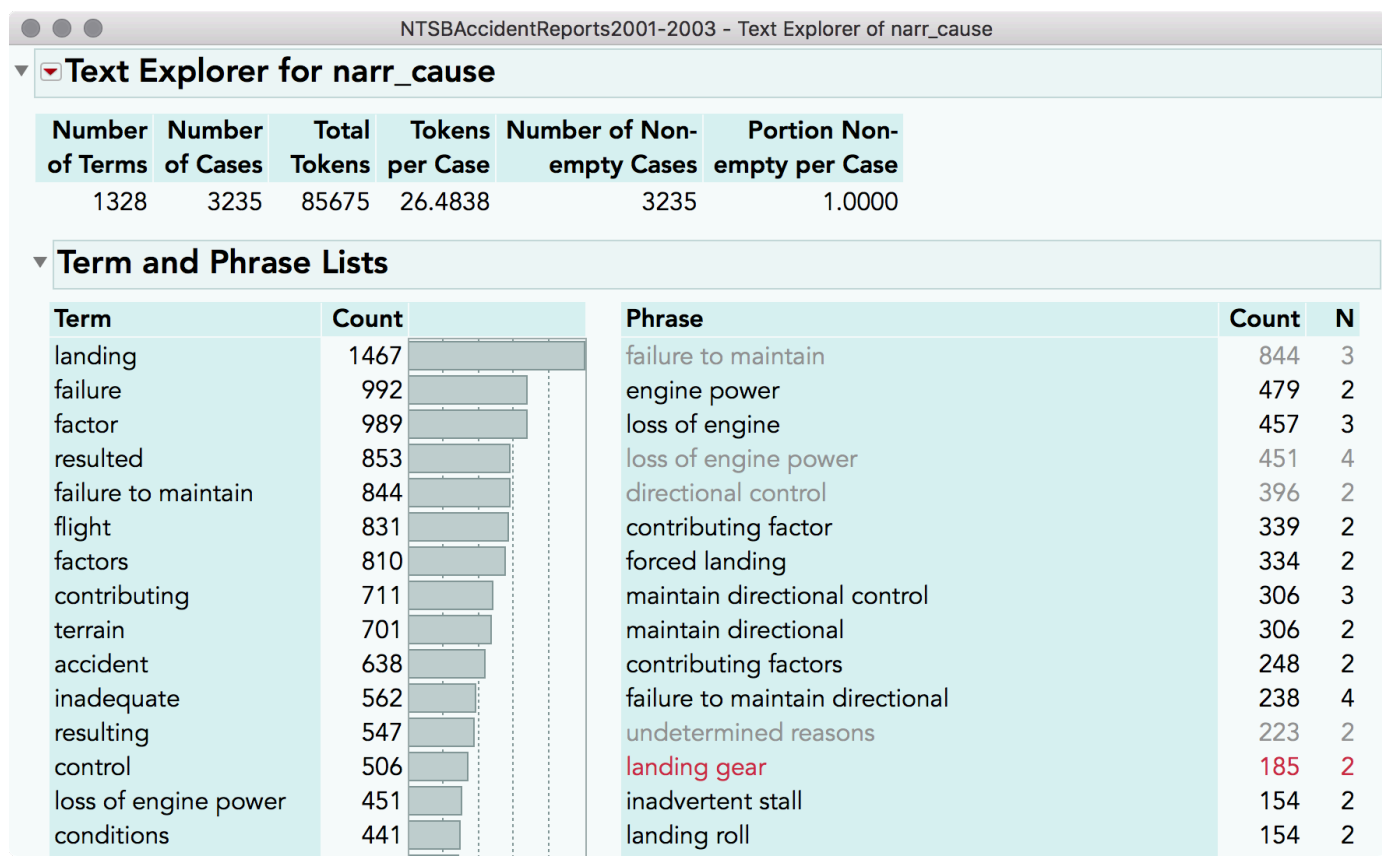

Fig. 3: Top terms and phrases after removing some terms and adding phrases

The term list can be visualized by a word cloud (Fig. 4). Top terms and phrases (added to the term list) are emphasized by font size. Everywhere where you see a term or phrase (e.g. in Fig. 3 and Fig. 4) you can right-click and choose "Show Text" to see the contextual information. This is very helpful if you want to understand how a single term or phrase is used.

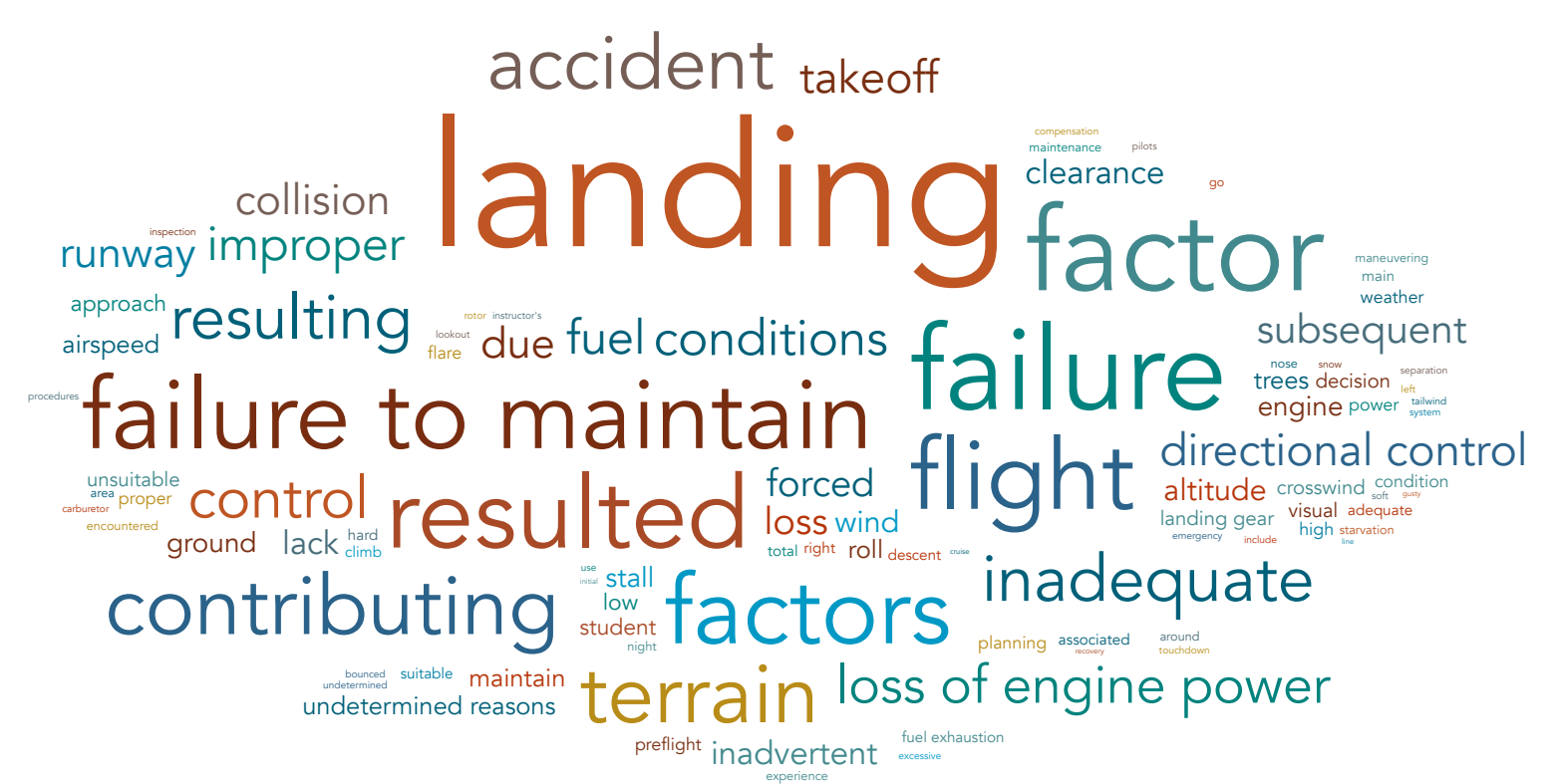

Fig. 4: Word Cloud 


\section{PART II: TEXT ANALYSIS}

Figure 5 shows the results from the dimension reduction in the document space (left, DTM rows) and term space (right, DTM columns), called Latent Semantic Analysis. Similar to Principal Component Analysis in multivariate analysis, Singular Value Decomposition (SVD) is used in text analysis to take advantage of the sparse nature of the DTM. With only two dimensions both related documents and terms group nicely. Results like SVD vectors or SVD Matrix can be exported for further analysis by other JMP platforms.
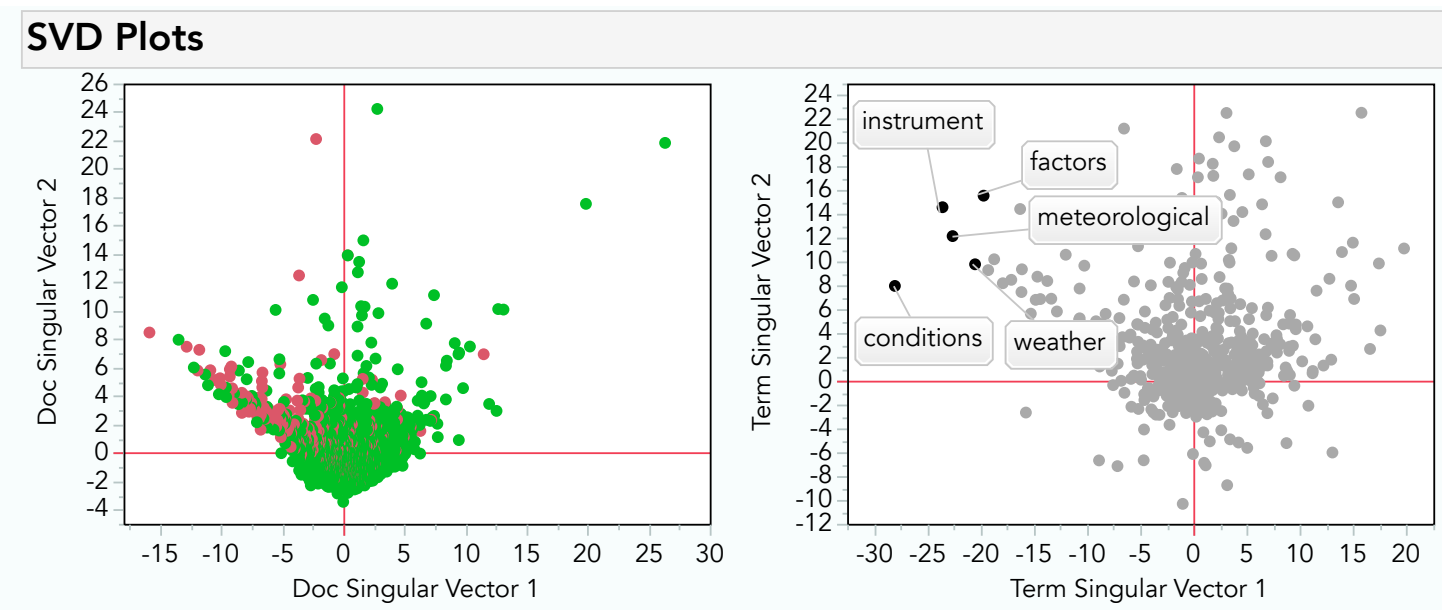

Fig. 5: Two-dim. document and term vector spaces after Latent Semantic Analysis (SVD)

Topic Analysis performs a varimax rotated singular value decomposition of the DTM to produce groups of terms called topics. This corresponds to Factor Analysis in multivariate analysis. In this example, Topic1 seems to represent the weather theme, while Topic6 is about maintenance.

Documents can be visually selected based on their topic scores, and more detailed relationships can be discovered by a combined scatterplot matrix of the rotated SVD vectors (topics) in the term and document space.

\begin{tabular}{|c|c|c|c|c|c|c|c|c|c|c|}
\hline \multicolumn{11}{|l|}{ Topic Words } \\
\hline \multicolumn{2}{|c|}{ Topic1 } & \multicolumn{2}{|c|}{ Topic2 } & \multicolumn{3}{|c|}{ Topic3 } & \multicolumn{2}{|c|}{ Topic4 } & \multicolumn{2}{|c|}{ Topic5 } \\
\hline Term & Score & Term & Score & Term & & Score & Term & Score & Term & Score \\
\hline conditions & 0.26629 & n89803 & 0.42364 & forced & & 0.2690 & midair & 0.36219 & directives & 0.32031 \\
\hline instrument & 0.26562 & rolling & 0.41177 & fuel & & 0.2547 & way & 0.34063 & local & 0.28624 \\
\hline meteorological & al 0.25200 & set & 0.31753 & loss of engin & ne power & 0.2336 & yield & 0.34063 & controller & 0.26957 \\
\hline continued & 0.21365 & cockpit & 0.30131 & starvation & & 0.1981 & atc & 0.27992 & faa & 0.25078 \\
\hline vfr & 0.21248 & insure & 0.26164 & suitable & & 0.1819 & lower & 0.24830 & follow & 0.23959 \\
\hline weather & 0.20342 & front & 0.25694 & due & & 0.1674 & lookout & 0.24036 & procedures & 0.23777 \\
\hline factors & 0.19526 & forward & 0.23763 & terrain & & 0.1610 & airplanes & 0.22820 & taxiing & 0.20765 \\
\hline flight & 0.19308 & brakes & 0.21348 & fuel exhausti & tion & 0.1498 & radio & 0.22003 & cross & 0.16843 \\
\hline fog & 0.18825 & attention & 0.21054 & unsuitable & & 0.1461 & visual & 0.21812 & md & 0.15902 \\
\hline ceilings & 0.17450 & parked & 0.20675 & failure to ma & aintain & -0.1445 & extra & 0.19409 & tower & 0.15873 \\
\hline imc & 0.16480 & colliding & 0.18631 & lack & & 0.1323 & instructions & is 0.17031 & company & 0.13894 \\
\hline night & 0.16343 & & & preflight & & 0.1258 & collision & 0.15169 & personnel's & 's 0.13476 \\
\hline adverse & 0.15879 & & & selector & & 0.1253 & & & & \\
\hline low & 0.15622 & & & tank & & 0.1227 & & & & \\
\hline dark & 0.15023 & & & & & & & & & \\
\hline \multicolumn{2}{|l|}{ Topic6 } & \multicolumn{2}{|c|}{ Topic7 } & \multicolumn{2}{|c|}{ Topic8 } & \multicolumn{3}{|c|}{ Topic9 } & \multicolumn{2}{|c|}{ Topic10 } \\
\hline Term & Score & Term & Score & Term & Score & Term & & Score & Term & Score \\
\hline maintenance & 0.21821 & pattern & 0.37247 & supervision & 0.26775 & stall & & 0.2194 & runway & 0.21757 \\
\hline personnel & 0.20892 & entry & 0.28841 & remedial & 0.23798 & airspeec & & 0.2098 & go & 0.17635 \\
\hline main & 0.20867 & traffic & 0.28284 & action & 0.22390 & landing & & -0.2008 & proper & 0.17585 \\
\hline landing gear & 0.20566 & using & 0.26881 & instructor's & 0.21273 & altitude & & 0.1692 & around & 0.17463 \\
\hline service & 0.16827 & without & 0.25605 & instructor & 0.21045 & compen & sation & -0.1453 & point & 0.16389 \\
\hline company & 0.15817 & executing & 0.25288 & student & 0.19544 & inadvert & & 0.1433 & factors & 0.15093 \\
\hline bulletin & 0.15699 & insure & 0.24649 & student's & 0.18438 & roll & & -0.1412 & touchdown & 0.14621 \\
\hline right & 0.15016 & manual & 0.24269 & dual & 0.18007 & adequa & & 0.1310 & wrong & 0.12354 \\
\hline actuator & 0.14899 & information & 0.23415 & certified & 0.16579 & crosswin & & -0.1297 & end & 0.12053 \\
\hline left & 0.13669 & correct & 0.22607 & inadequate & 0.16435 & low & & 0.1286 & accident & 0.11897 \\
\hline inspection & 0.13135 & pilots & 0.22601 & cfi & 0.15321 & wind & & -0.1271 & attain & 0.11781 \\
\hline collapse & 0.12798 & operating & 0.19662 & delayed & 0.14908 & direction & hal control & -0.1269 & tailwind & 0.11619 \\
\hline assembly & 0.12746 & adequately & 0.17632 & flight & 0.13495 & spin & & 0.1201 & decision & 0.11271 \\
\hline attach & 0.12503 & & & & & conditio & & -0.1102 & overrun & 0.11254 \\
\hline
\end{tabular}

Fig. 5: Topic Words for 10 topics 
Other analysis options include Clustering Analysis of terms and documents. The number of clusters can be set and cluster membership can be saved to the existing (Cluster Docs) or a new data table (Cluster Terms).

\section{PART III: DISCOVER RELATIONSHIPS AND PREDICTIVE MODELING}

The Text Explorer top triangle menu (Fig. 2, right) shows several options for saving the DTM or any analysis results. This allows you to explore relationships between your unstructured and structured data, or to use numeric representations of your text data as independent variables in predictive modeling.

The result from the term creation phase, the DTM, can be directly added to the data table. The number of terms can be chosen in order to reduce the dimensionality of the matrix. An interesting option is the type of weighting factor stored in indicator columns: Compared to 'Binary' (term is used or not), settings like 'TF-IDF' (Term-Frequency Inverse-Document-Frequency) can be very powerful, see Help Text Explorer (2017).

Figure 6 shows all documents over time (X-axis) and split by 'fatal' (Y-axis) and cluster 110 assigned by Cluster Documents. This result suggests for instance to investigate some special cases like Cluster 4 or 8 . For cluster 9 fatality could be reduced over time.

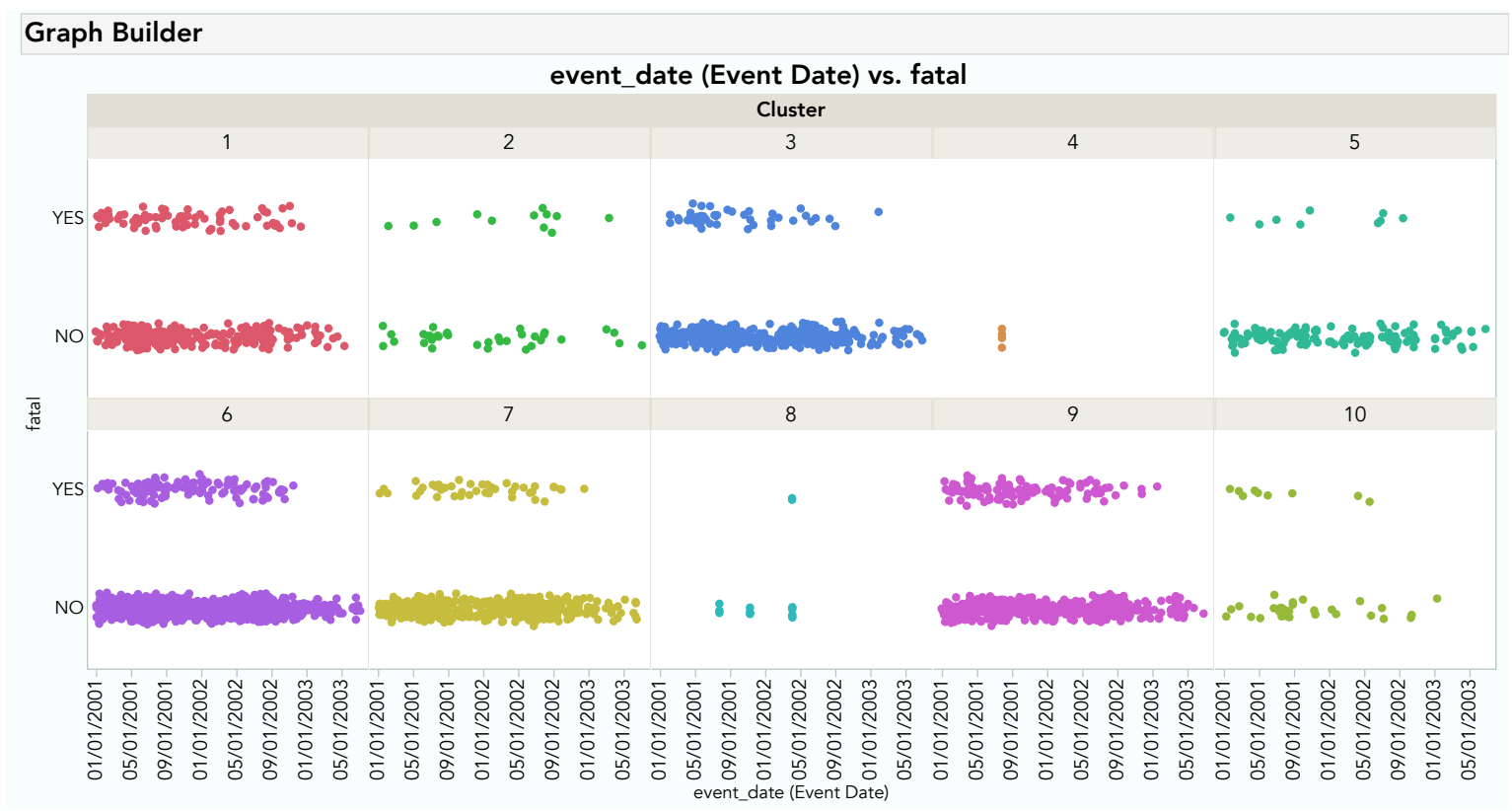

Fig. 6: Combining text analysis results (here wrap by 'Cluster' from Cluster Documents) with other data (Here $\mathrm{Y}=$ 'fatal' and $\mathrm{X}=$ ='event_date')

Figure 7 shows an example for a predictive model solely based on text information: Using the Generalized (Penalized) Regression personality in Fit Model, we built a model predicting 'fatal' using all(!) indicator variables from the DTM. The LASSO method helps with variable selection: By moving the red slider on the left towards zero, the penalty is increased and more variables are removed from the model. The validation plot on the right helps to select the "best" model, here based on AIC corrected.

The model tells us that the term 'landing' (with binary weights) is highly significant and negative. Probably this means that we don't need to worry too much about fatal accidents during the landing phase.

A next step could be to improve the model by adding structured data which is also available, maybe about time or weather conditions. All of this takes you minutes with JMP, rather than days of programming and tedious text processing. Interactive visual outputs are made directly available to communicate your findings. 

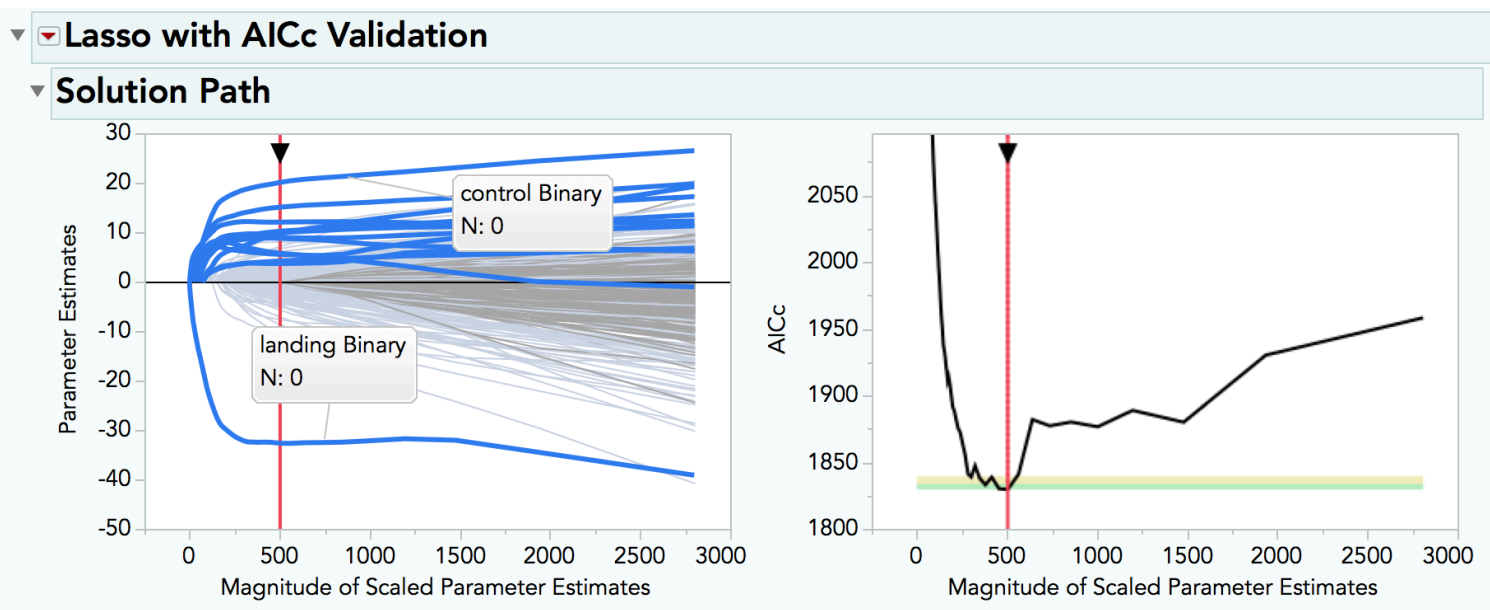

\begin{tabular}{|c|c|c|c|c|c|c|c|}
\hline Term & Estimate & Std Error & $\begin{array}{r}\text { Wald } \\
\text { ChiSquare }\end{array}$ & $\begin{array}{r}\text { Prob > } \\
\text { ChiSquare }\end{array}$ & Lower $95 \%$ & Upper 95\% & $\begin{array}{l}\text { Singularity } \\
\text { Details }\end{array}$ \\
\hline Intercept & -2.507953 & 0.134383 & 348.29741 & $<.0001^{*}$ & -2.771339 & -2.244567 & \\
\hline landing Binary & -1.181923 & 0.1660677 & 50.653357 & $<.0001 *$ & -1.50741 & -0.856437 & \\
\hline failure Binary & 0 & 0 & 0 & 1.0000 & 0 & 0 & \\
\hline factor Binary & -0.093249 & 0.1279109 & 0.5314684 & 0.4660 & -0.34395 & 0.1574514 & \\
\hline resulted Binary & 0 & 0 & 0 & 1.0000 & 0 & 0 & \\
\hline failure to maintain Binary & 0.3893494 & 0.1764678 & 4.8679732 & $0.0274^{*}$ & 0.0434789 & 0.7352198 & \\
\hline flight Binary & 0.3782425 & 0.1545093 & 5.9928205 & $0.0144^{*}$ & 0.0754098 & 0.6810751 & \\
\hline factors Binary & 0 & 0 & 0 & 1.0000 & 0 & 0 & \\
\hline contributing Binary & 0 & 0 & 0 & 1.0000 & 0 & 0 & \\
\hline terrain Binary & 0.0162515 & 0.1870853 & 0.0075458 & 0.9308 & -0.350429 & 0.382932 & \\
\hline
\end{tabular}

Fig. 7: Using text analysis results in predictive modelling (here: Generalized Regression)

\section{CONCLUSION}

Making discoveries and visualizing information from unstructured text data has never been easier and more fun. Using version 13 of JMP (and of JMP Pro for even more powerful text analysis) allows you to add unstructured text data to your data mining scenario. Combining the results from your text analysis with other structured data can take your predictive models to the next level.

Ideas for future developments include more options to import text data into JMP (see also JMP Addin, 2016), adding other data formats like audio or video and to further streamline the integration of text analysis and predictive modeling tools.

\section{REFERENCES}

JMP Addin - Adsurgo TM Tools (2016): Text Importer - Text, PDF, Word Documents, and Powerpoint. JMP Community. http://bit.ly/2srow3s

JMP Help Text Explorer (2017): Text Explorer - Explore Unstructured Text in Your Data. http://bit.ly/2rh2vok

JMP Home (2017): http://www.JMP.com

Karl A., Wisnowski J., Rushing W.H. (2015). A practical guide to text mining with topic extraction. WIREs Comput Stat 2015, 7:326-340. doi: 10.1002/wics.1361

Klimberg, Ron and B.D. McCullough (2016). Fundamentals of Predictive Analytics with JMP, Second Edition. Cary, NC: SAS Institute Inc. Chapter 15: Text Mining.

Singh V. and Zhang Q. (2016). Text Mining Online Reviews with JMP 13. JMP Discovery Summit, Cary, NC: http://bit.ly/2t5CWTs

Vijayan, J. (2015). Solving the Unstructured Data Challenge. CIO.

http://www.cio.com/article/2941015/big-data/solving-the-unstructured-data-challenge.html 